\title{
VIVÊNCIA DE ESPIRITUALIDADE/RELIGIOSIDADE E QUALIDADE DE VIDA EM PACIENTES EM TRATAMENTO HEMODIALÍTICO
}

\section{EXPERIENCE OF SPIRITUALITY/RELIGIOSITY AND QUALITY OF LIFE IN PATIENTS IN HEMODIALYTIC TREATMENT}

\author{
Glaucirene Siebra Moura Ferreira ${ }^{1 \# *}$ Francisco Mayron Morais Soares ${ }^{2} *$ Rodrigo da Silva $^{*}$ \\ Nunes $^{3}$ * Paciolo Montini da Costa Oliveira ${ }^{4}$ * Rejânio de Aguiar Araújo * \\ Joelma de Oliveira Ripardo ${ }^{*}$ José Gerardo da Silva $^{6} *$ Paula Frassinnetti Camurça Castelo \\ Branco Fernandes ${ }^{7}$
}

\section{RESUMO}

Objetivo: apontar quais sentimentos influenciam a vivência da espiritualidade/religiosidade e qualidade de vida dos pacientes em tratamento hemodialítico. Método: Estudo exploratório/descritivo, com abordagem qualitativa utilizando o Software IRAMUTEQ 0.5 com uso da análise de similitude e nuvem de palavras, incluindo 192 pacientes portadores de DRC, em hemodiálise, no período de setembro a novembro de 2019. Resultados: Árvore da similitude mostra os sentimentos e sensações antes, durante e depois das sessões de hemodiálise: fraco; dor; sentimento de preocupação; não dorme; hipertensão; acostumado; preocupado; cochila; tem ansiedade; aliviado; tontura; medo; tranquilo; quer ir para casa: vontade de sair; passar. Papel da Espiritualidade na nuvem de palavras destacam-se: Importante; força; vida; Deus; fé, ajuda; bem. $\mathrm{Na}$ vivência da religiosidade e espiritualidade encontramos: Missa; oração; dia de orar; rezar; vivenciar; só Deus; frequentar; fé; crer; ler; televisão, família. Concluímos: que: A religiosidade apresentou fonte de conforto, esperança e segurança nos pacientes com DRC e promoveu o bemestar físico e mental, com auxilio no enfretamento da doença.

Palavras-chave: Espiritualidade; Religiosidade; Qualidade de Vida; Doença Renal Crônica; Hemodiálise.

\begin{abstract}
Objective: to point out which feelings influence the experience of spirituality/religiosity and quality of life of patients undergoing hemodialysis. We searched for the methodology: qualitative descriptive using the IRAMUTEQ 0.5 Software with the use of similarity analysis and word cloud, including 192 patients with CKD on hemodialysis, from September to November 2019. Results: Similarity tree shows feelings and sensations before, during and after hemodialysis sessions: weak; ache; feeling of worry; do not sleep; hypertension; accustomed; worried; nap; have anxiety; relieved; dizziness; fear; quiet; want to go home: want to go out; pass. Role of Spirituality in the word cloud stand out: Important; force; life; God; faith, help; well. In the experience of religiosity and spirituality we find: Mass; prayer; day to pray; to pray; experience; Only god; attend; faith; believe; to read; television, family. We concluded: that: Religiosity presented a source of comfort, hope and security in patients with CKD and promoted physical and mental well-being, helping to cope with the disease.
\end{abstract}

Keywords: Spirituality; Religiosity; Quality of Life; Chronic Kidney Disease; Hemodialysis.

\footnotetext{
1 \#https://orcid.org/0000-0002-1742-2096

2 https://orcid.org/0000-0001-7316-2519

3 https://orcid.org/0000-0001-7873-5451

4 https://orcid.org/0000-0003-4046-5841

5 https://orcid.org/0000-0002-9774-510X

6 https://orcid.org/0000-0002-9774-510X

7 https://orcid.org/0000-0002-7442-301X
} 


\section{INTRODUÇÃO}

A religiosidade é entendida como o ritual ou prática, através da qual os indivíduos expressam a sua ligação ao Transcendente, de forma individual e/ou coletiva, através de uma organização, que pode ser distinta. Pode ser entendida como uma religiosidade intrínseca, quando a religião é procurada pelos indivíduos como um fim em si mesmo, e extrínseca, quando esta busca se dá para atingir outros fins ${ }^{(1)}$.

Para se distinguir de um mero ritual, alguns autores diferenciam a espiritualidade da religiosidade. $\mathrm{O}$ conceito de espiritualidade versa pelo menos duas perspectivas diferentes. Na primeira, a espiritualidade é compreendida como uma busca pelo significado da vida e o relacionamento desta com o sagrado/transcendente. No segundo entendimento, a espiritualidade se refere à manifestações humanas que buscam a superação de si, ou de obstáculos, no qual não há necessariamente uma ligação com o sagrado $^{(2)}$.

Nos últimos anos tem-se identificado estudos que reconhecem o resultado da religiosidade/espiritualidade relativas à saúde enquanto recurso para confronto de dificuldades nos processos de adoecimentos. Portanto, estas são dimensões que influenciam desde o processo diagnóstico e adesão ao tratamento, à terapêutica e a convivência diária $^{(3)}$.
O convívio com a doença renal crônica e o doloroso tratamento geram conflitos existenciais nos pacientes, suscetíveis de provocar angústia espiritual podendo agravar os sintomas físicos e emocionais e a capacidade para enfrentar a $\operatorname{doença~}^{(4)}$.

Deste modo, estudo ${ }^{(5)}$ afirma que a espiritualidade é um domínio considerável da qualidade de vida, sobretudo nos pacientes terminais, onde a vida é ameaçada pelas doenças crônicas. De acordo com os autores, para muitas pessoas, espiritualidade e religião é uma fonte que ajuda e contribui para o bemestar e o enfrentamento das dificuldades frequentes da vida.

Convém lembrar que, o Tratamento da Doença Renal Crônica (DRC), na maioria das vezes, gera no paciente frustração e limitações, uma vez que é acompanhada de diversas restrições, e modificações no seu cotidiano e no desempenho de suas atividades laborais ou mesmo em suas relações sociais, pois devido a esse tratamento ser responsável por muitas mudanças no cotidiano do indivíduo, alguns processos podem refletir na esperança de vida do mesmo. Porém, manter a esperança perante a doença é um processo contínuo e importante, pois estimula o indivíduo e a família a buscar novos caminhos ou aceitação da nova condição imposta pela doença. Nesse contexto, a espiritualidade e a religiosidade também estão associadas como ferramentas de apoio e fortalecimento, para o 
enfrentamento das dificuldades no cotidiano, relacionadas às suas crenças espirituais individuais ${ }^{(6)}$.

No entendimento de Sartore e Gross ${ }^{(7)}$ o estado espiritual dos pacientes que vivem com a DRC e passa pelo tratamento de hemodiálise influência o enfrentamento da doença, sendo um fator atuante nesse processo. Dessa forma, é importante que os profissionais de saúde atentem aos aspectos relacionados à manifestação da espiritualidade, pois a ausência da mesma pode agravar os sintomas físicos e emocionais e diminuir a capacidade de enfrentar a doença.

A propósito das afirmações supracitadas, necessita-se também de se ter uma compreensão no que se refere à religião enquanto escolha individual, pois, esta supõe uma afinidade com diversas práticas religiosas e não necessariamente uma proximidade teológica, esta faz sentido e existe onde se encontra uma comunidade, disponibilizando aos indivíduos significado da vida para além da realidade terrena e possibilitando explicações para fatos misteriosos da vida como a morte $\mathrm{e}^{(8-3)}$.

Neste sentido, Gergianaki ${ }^{(9)}$ afirmam que há um efeito positivo em discutir a espiritualidade nos cuidados de saúde e esse resultado tem sido explorado e ratificado em várias doenças crônicas, deste modo, tem-se vários estudos sugerindo que abordagens espirituais podem ser úteis para melhorar a saúde mental e estado social dos pacientes. Porém, lamenta-se que os profissionais de saúde muitas vezes não estão conscientes da importância da espiritualidade para os pacientes com doenças crônicas, talvez, devido à falta de tempo ou de habilidades adequadas, não abordem e não incorporem adequadamente esta prática em sua rotina clínica.

Por entender esse papel importante da prática religiosa/espiritual como possíveis fatores intervenientes no processo saúdedoença nos interessamos em abordar essa relação em uma condição crônica específica que é a doença renal crônica. Em nossa experiência temos observado que os pacientes com doença renal crônica passam pelo duplo desafio de se adaptar à doença e ao tratamento, já que há significativas transformações fisiológicas, psicossociais e espirituais que decorrem dessa condição. Vemos também que em meio a essas adversidades, muitos pacientes se apoiam na fé e na religião como forma de encontrar apoio e alívio para seu sofrimento.

Desse modo, o objetivo deste trabalho é sobre a relação espiritualidade/religiosidade e Qualidade de Vida a condição crônica da doença renal. Assim, pretendemos contribuir com a clínica e a gestão de serviços destinados a este público, de modo a traçar diferentes estratégias que possam subsidiar intervenções e modos de atenuar o sofrimento, promovendo maior qualidade de 
vida para as pessoas que vivenciam esta situação.

\section{MÉTODO}

Tratou-se de um estudo exploratório descritivo, com abordagem qualitativa. A análise qualitativa foi realizada com o software Iramuteq 0.7 com uso da análise de similitude e nuvem de palavras, incluindo 192 pacientes portadores de DRC, em hemodiálise, no período de setembro a novembro de 2019.

A pesquisa foi realizada nos dois maiores centros de diálise da macrorregião noroeste do Estado, Santa Casa de Misericórdia de Sobral e no Centro de Nefrologia de Itapipoca (CENIT). Estabeleceu-se como critérios de inclusão: pacientes portadores de DRC, em hemodiálise, maiores de 18 anos e de ambos os sexos. Foram excluídos pacientes que estavam internados no momento da coleta, que evoluíram para o óbito nesse período e que tinham iniciado a hemodiálise até três meses antes da realização do estudo. A coleta de dados ocorreu por meio de entrevistas individuais aos pacientes durante os atendimentos de hemodiálise, aplicada pelos pesquisadores. Para execução das entrevistas foram utilizados os seguintes instrumentos: questionário sociodemográfico e clínico, o Kidney Disease and Quality of Life ShortForm (KDQOL-SF TM) e Escala de Espiritualidade e Religiosidade da Organização Mundial de Saúde-OMS.
Para mensurar a religiosidade e espiritualidade foi utilizado o questionário WHOQOL-SRPB, o qual faz parte do instrumento de Avaliação da Qualidade de Vida da OMS, desenvolvido por um grupo de especialistas na área que se reuniu e elaborou uma síntese dos principais aspectos da dimensão espiritual. Este instrumento contém 32 itens, dividido em oito facetas: conexão com ser ou força espiritual, sentido na vida, admiração, totalidade/integração, força interior, paz interior/serenidade/harmonia, esperança/otimismo, fé. $\mathrm{O}$ escore de pontuação para cada item de cada faceta varia de 01 a 05 ponto.

$\mathrm{O}$ instrumento foi validado e apresentou boas qualidades psicométricas para uso no Brasil No entanto, os autores aconselham novos estudos com populações específicas, como diferentes religiões, grupos culturais e/ou doenças. Deste modo, para a presente pesquisa o instrumento mostra-se ideal para compreender como as pessoas com DRC lidam com o enfrentamento da doença utilizando a religiosidade e a espiritualidade ${ }^{(10-11)}$.

$\mathrm{O} \quad \mathrm{KDQOL}-\mathrm{SF}^{\mathrm{TM}}$, instrumento validado no Brasil para mensurar a qualidade de vida nos indivíduos com DRC, possui 80 itens, o Short Form Health Survey (SF-36) e mais 43 itens sobre DRC. O SF-36 é fragmentado em oito dimensões: funcionamento físico (dez itens); limitações causadas por problemas de saúde física 
(quatro itens); limitações causadas por problemas de saúde emocional (três itens); funcionamento social (dois itens); saúde mental (cinco itens); dor (dois itens); vitalidade (quatro itens); percepções de saúde geral (cinco itens) e estado de saúde atual em comparação com um ano atrás (um item). Os itens relacionados à doença renal estão divididos em 11 dimensões: sintomas / problemas (12 itens); efeitos da doença renal na vida diária (oito itens); sobrecarga imposta pela doença renal (quatro itens); condição de trabalho (dois itens); função cognitiva (três itens); qualidade das interações sociais (três itens); função sexual (dois itens); sono (quatro itens); escala de suporte social (dois itens); escala de estimulação da equipe de diálise (dois itens) e escala de satisfação do paciente (um item) Para essa investigação foram escolhidos apenas oito itens que mais interfere nas atividades de vida diária dos pacientes com DRC.

Os dados do instrumento KDQOL$\mathrm{SF}^{\mathrm{TM}}$ foram colocados numa planilha do programa Excel for Windows disponibilizada on-line pela RAND Health Care (https://www.rand.org/health-

care/surveys tools/kdqol.html), que mede automaticamente as pontuações por itens e dimensões de todo o instrumento. Não havendo ponto de corte nesse instrumento, utilizou-se a média de respostas expostas pelos pacientes no intuito de comparar com os resultados de outras pesquisas sobre a temática, que foram publicadas anteriormente. O protocolo do estudo foi analisado e aprovado pelo Comitê de Ética, preconizado pela Resolução No 466/12 do Ministério da Saúde, no Brasil (Protocolo $\mathrm{n}^{\circ}$ 3.375.367/ UNINTA).

\section{RESULTADOS}

Figura 1 - Frequência de palavras conforme amostra. Fortaleza, Ceará, Brasil, 2021.

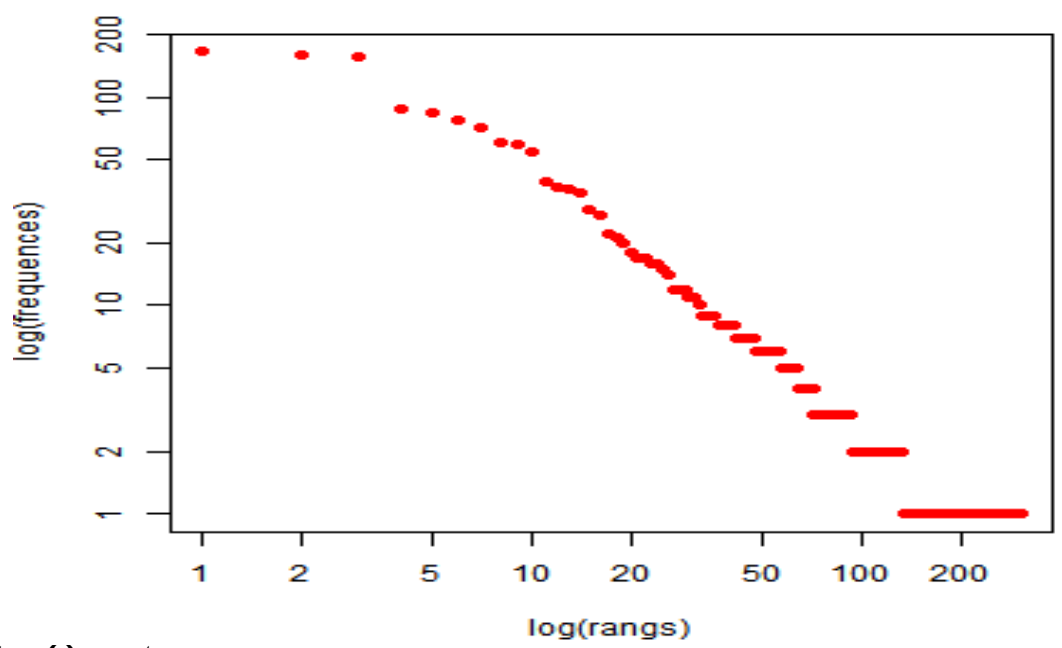

Fonte: Us autores. 
Figura 2 - Árvore da similitude conforme amostra os sentimentos e sensações antes, durante e depois das sessões de hemodiálise. Fortaleza, Ceará, Brasil, 2021.
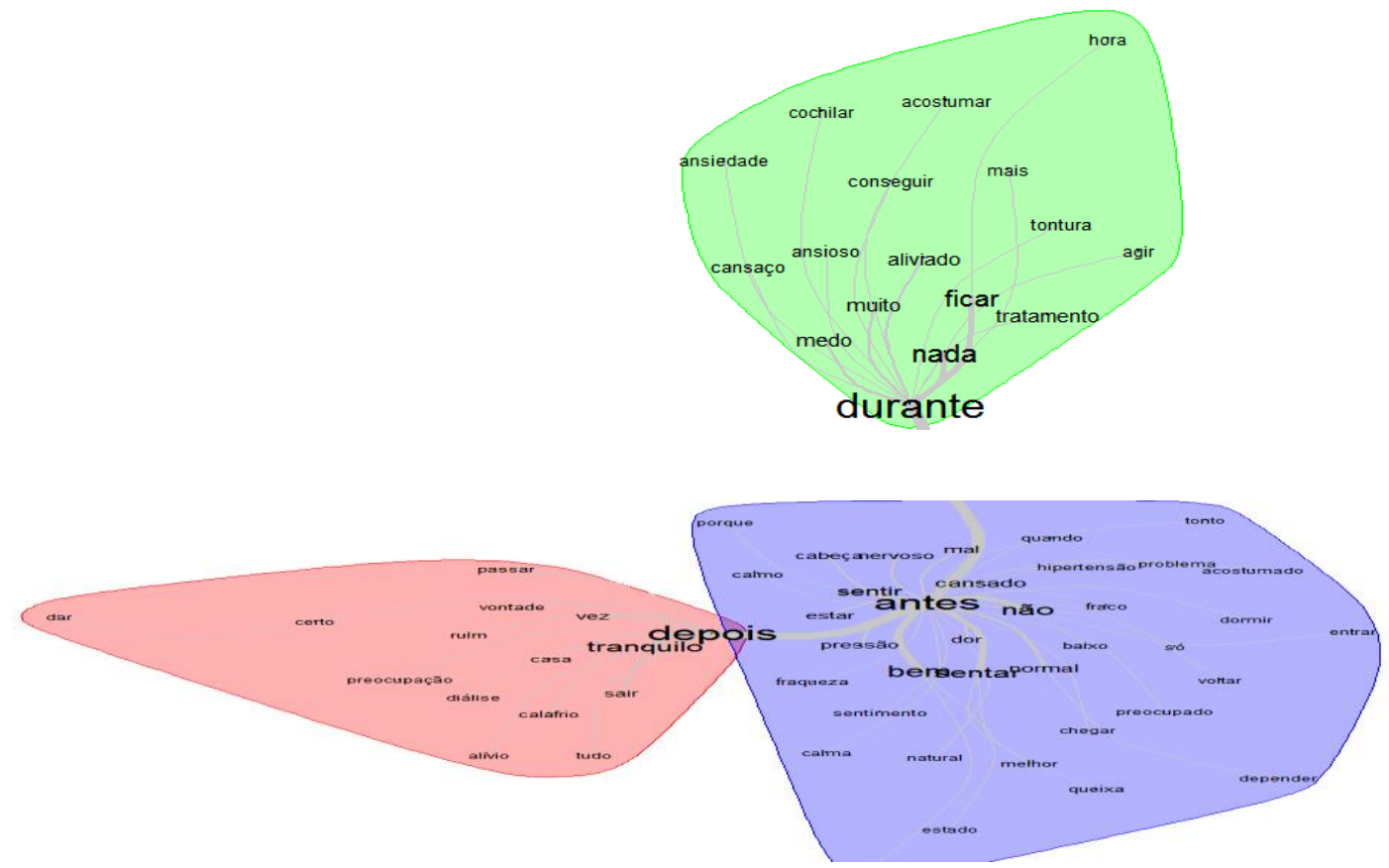

Fonte: Os autores.

Figura 3 - Papel da Espiritualidade conforme amostra. Fortaleza, Ceará, Brasil, 2021.

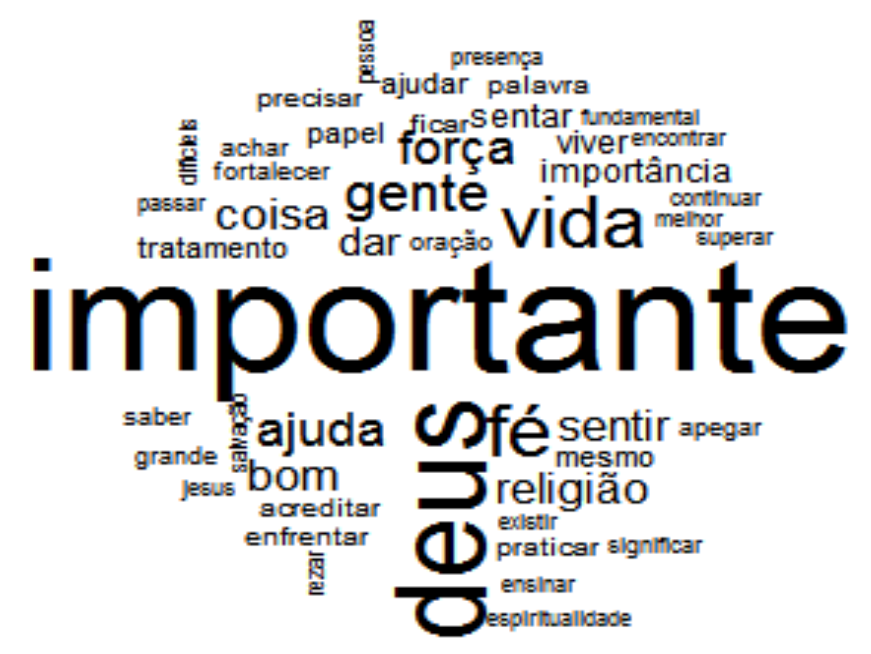

Fonte: Os autores. 
Figura 4 - Arvore da similitude em relação ao Papel da Espiritualidade conforme amostra. Fortaleza, Ceará, Brasil, 2021

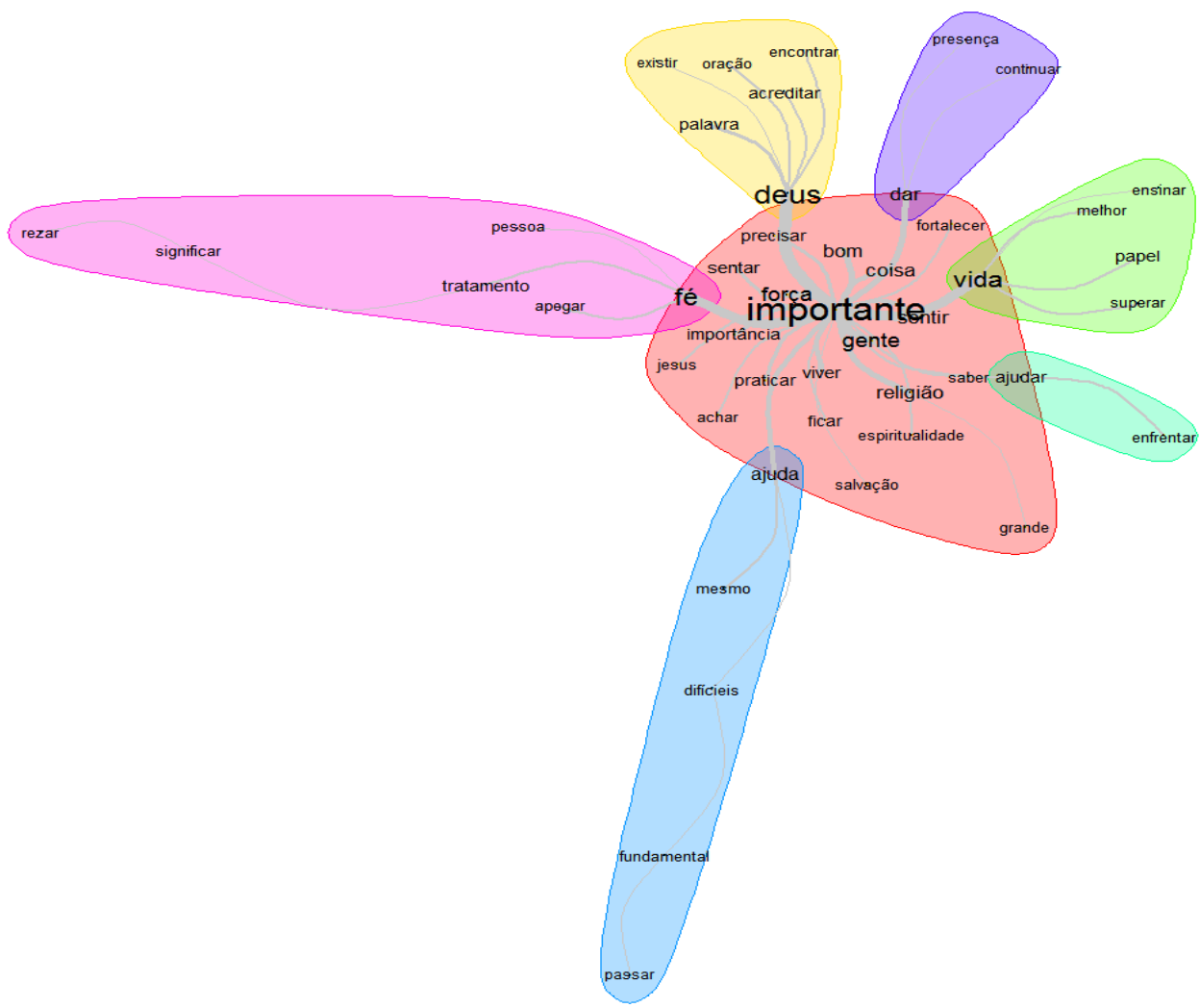

Fonte: Os autores.

Figura 5 - Vivência da religiosidade e espiritualidade conforme amostra. Fortaleza, Ceará, Brasil, 2021.

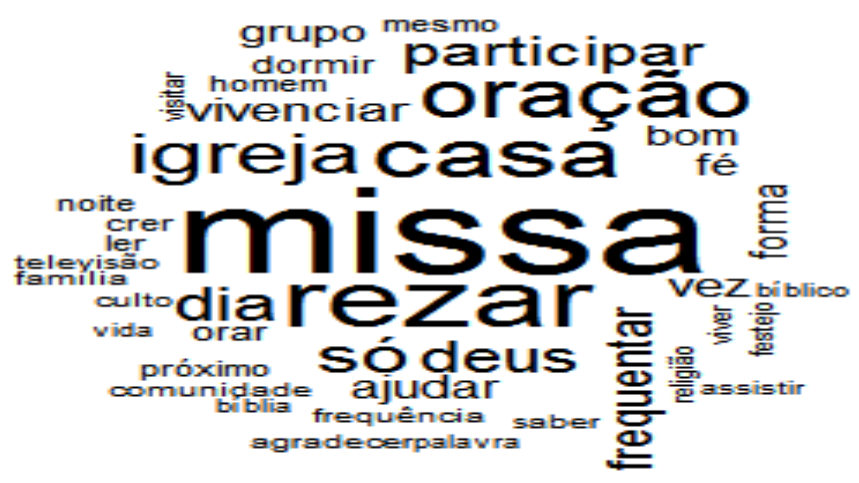

Fonte: Os autores. 


\section{DISCUSSÃO}

\section{Religiosidade, Espiritualidade e Crença}

No que diz respeito a ciências da saúde, os estudos atestam sobre a influência que a religião pode ter no processo saúde/doença e destacam sua importância enquanto uma forma de enfrentamento na experiência de tal processo ${ }^{(12-13)}$.

A religião ou crenças e práticas religiosas podem ser determinantes do processo saúde-doença na medida em que prega a adoção de hábitos e de comportamentos saudáveis que beneficiam aqueles que os praticam. Algumas práticas religiosas trazem efeitos salutares de saúde física e mental. Estas práticas geram reflexões nos membros da família e, por vezes, têm um papel importante na prevenção de doenças ${ }^{(14)}$.

Assim, a religião e espiritualidade têm se tornado cada vez mais relevantes no processo saúde-doença, nos cuidados à saúde dos indivíduos e tem sido vista como um intenso protótipo a ser constituído no exercício cotidiano dos trabalhadores da área da saúde. A religiosidade e a espiritualidade são extremamente importantes na prestação de cuidados terapêuticos e pautados na dor, com o intuito de abranger a totalidade na assistência à saúde do ser humano, uma vez que o método religioso jamais precisa substituir o método médico, mais sim complementá-1o ${ }^{(15)}$.
Caires ${ }^{(16)}$ relata que a religiosidade e a espiritualidade atentam para o fato de se empenhar na constituição desta nova questão explanada para o paciente, fazendo com que ele procure entender a própria patologia, a aflição, a morte e a vivência.

Segundo $\mathrm{Melo}^{(17)}$ vale salientar a escassez de pesquisas desenvolvidas com a população renal crônica sobre a relação desses aspectos e pontuar que os achados da presente pesquisa contribuem para ampliar o conhecimento nesta área. Ficou evidente, a importância de estudar estes fenômenos para as práticas de saúde, o que reitera a necessidade de valorizá-los e incluí-los nas formações profissionais, com destaque, neste caso, aos profissionais de enfermagem que atuam diretamente no cuidado.

De acordo com Diniz ${ }^{(18)}$ os termos espiritualidade e religiosidade possuem papel no que se refere à atribuição de significados à vida, como um próprio recurso para construir a esperança de vida, especialmente no tocante ao enfrentamento de doenças que causam mudanças no estado clínico e físico dos pacientes, como nos casos de hemodiálise.

Para Bravin ${ }^{14)}$ os benefícios da espiritualidade e/ou religiosidade na prática clínica têm sido evidenciados. A espiritualidade, religiosidade e a saúde de pacientes dialíticos apontaram melhorias na relação entre médico-paciente, na qualidade 
de vida e no enfrentamento da doença. Assim, aproximar-se de Deus, da igreja e de seus membros resulta em maior apoio emocional e social.

\section{Distribuição de Qualidade de vida}

O termo qualidade de vida consiste em uma ampla gama de conceitos que afetam vários domínios da vida, como boa saúde, moradia satisfatória, emprego, segurança, educação e lazer. Quando ela é relacionada à saúde da pessoa, levam-se em conta os aspectos físicos, sociais e emocionais causados pela doença ou pelo tratamen ${ }^{(19)}$.

Segundo Grandizoli ${ }^{(20)}$ a qualidade de vida dos indivíduos com DRC tem sido motivo de atenção, pois a percepção de melhora ou piora pode ajudar no tratamento da doença. Acredita-se que a DRC e o tratamento hemodialítico interferem diretamente na percepção do paciente frente ao suporte social recebido, à sua $\mathrm{QV}$, pois esta abarca as limitações físicas e as alterações na vida cotidiana. Assim que é diagnosticado, o paciente precisa aprender a conviver com o novo dia a dia e com suas diversas limitações, pois sua rotina é intensamente modificada

O déficit na qualidade de vida e o tipo de tratamento ao qual o paciente renal crônico é submetido podem contribuir para o surgimento de doenças psicoafetivas, dentre elas, os transtornos de ansiedade e depressão, sendo os distúrbios psiquiátricos mais comuns em portadores de doença renal crônica ${ }^{(21)}$.

A DRC ou IRC gera ao paciente frustração e limitações, uma vez que é acompanhada de diversas restrições, entre elas a manutenção de uma dieta específica, associada às restrições hídricas, e a modificação na aparência corporal em razão da presença do cateter para acesso vascular ou da fístula arteriovenosa, fazendo com que ocorram mudanças na dinâmica de vida, adaptações a novos hábitos e comportamentos $^{(22)}$.

$\mathrm{O}$ tratamento por $\mathrm{HD}$, juntamente à progressão da DRC, causa limitações e prejuízos nos estados de saúde mental, física, funcional, bem-estar geral, interação social e satisfação de pacientes. A IRC e, em consequência, a HD, acabam por gerar muitas modificações na vida dos pacientes, o que a torna muitas vezes de difícil aceitação, pois além de ser um tratamento permanente e doloroso, acaba por limitar muitas atividades que davam prazer e autonomia ao indivíduo portador, como, por exemplo, o trabalho, a alimentação e o lazer ${ }^{(22)}$.

\section{CONSIDERAÇÕES FINAIS}

No presente estudo, existe uma influência da religiosidade na QV dos pacientes renais crônicos. Observou-se que a religiosidade e espiritualidade têm impacto positivo com domínios sociais, psicológicos e apresentam boa correlação. 
A religiosidade apresentou fonte de conforto, esperança e segurança nos pacientes com DRC e promoveu o bem-estar físico e mental, com auxílio no enfretamento da doença.

\section{REFERÊNCIAS}

1. Catre MNC, et al. O domínio SRPB (Spirituality, Religiousness and Personal Beliefs) do WHOQOL: O estudo com grupos focais para validação da versão em Português europeu do WHOQOL-SRPB. Aná. Psicológica. [Internet]. 2017 [acesso em 12 de ag 2021]; 32(4): 401-17. Disponível em: https://impactumjournals.uc.pt/rppedagogia/issue/view/ 275.

2. Forte S, Serbena CA, Scaduto AA. Mensuração da espiritualidade/religiosidade em saúde no Brasil: Uma revisão sistemática. Cien Saude Colet. [Internet]. 2018 [acesso em 12 de ago 2021]; 25(4):1463-1474. Doi: 10.1590/141381232020254.21672018

3. Tavares CQ, Valente TCO, Cavalcanti, APR. Carmos HO. Espiritualidade, religiosidade e saúde: velhos debates, novas perspectivas. Interações [Internet]. 2016 [acesso em 12 de ago 2021];11(20):85-7. Disponível em: http://periodicos.pucminas.br/index.ph p/interacoes/article/view/P.1983-

2478.2016v11n20p85

4. Gomes IC, et al. Atitudes frente à dor e à espiritualidade dos pacientes renais crônicos em hemodiálise. BrJP. [Internet]. 2018 [acesso em 12 de ago 2021]; 1,

(4):320-4. Doi.org/10.5935/2595-0118.20180061

5. Fradelos EC, Tzavella F, Koukia E, et al. A tradução, validação e adaptação cultural da avaliação funcional da doença crônica - bem-estar espiritual 12 (facit-sp12) escala em língua grega. Mater Sociomed. [Internet]. 2016 [acesso em 12 de ago 2021]; 28 (3): 229-34. Doi: 10.5455 / msm.2016.28.229-234

6. Paula ES, Nascimento LC, Rocha SMM. Religião e espiritualidade: experiência de famílias de crianças com Insuficiência Renal Crônica. Rev Bras Enferm. [Internet]. 2009 [acesso em 12 de ago 2021];62(1):100-106. Doi.org/10.1590/S003471672009000100015

7. Sartore ACA, Gross SAA. Escala de Esperança de Herth - instrumento adaptado e validado para a língua portuguesa. Rev Esc Enferm USP. [Internet]. 2008 [acesso em 14 de ago 2021];42(2):227-32. Disponível em: https://www.scielo.br/j/reeusp/a/XjXD h8mNS3bvR46q8Yrk7Bm/?lang=pt\&f ormat $=$ pdf

8. Gabatz C. Religiosidade Brasileira Contemporânea: Pluralismo, Secularização e Múltiplas Pertenças. In:

CONGRESSO

INTERNACIONAL DA FACULDADES EST, 2., 2014, São Leopoldo. Anais (on line) do Congresso Internacional da Faculdades EST. São Leopoldo: EST, v. 2, 2014. Disponível em: http://anais.est.edu.br/index.php/congr esso/article/download/256/218

9. Gergianaki I, Kampouraki M, Williams S, Tsiligianni I. Avaliando a espiritualidade: há um papel benéfico no manejo da DPOC? NPJ Prim Care Respir Med. [Internet]. 2019 [acesso em 12 de ago 2021]; 29: 23. Disponível em: https://www.ncbi.nlm.nih.gov/pmc/arti cles/PMC6538715/. 
10. Leimig MBC, Lira RT, Peres FB, Ferreira AGC, Falbo AR. Qualidade de vida, espiritualidade, religiosidade e esperança em pessoas com doença renal crônica em hemodiálise. Rev Soc Bras Clin Med. [Internet].2018 [acesso em 12 de ago 2021];16(1):30-6. Disponível em: https://docs.bvsalud.org/biblioref/2018 /06/884990/dezesseis_trinta.pdf

11. Oliveira APB, Schmidt DB, Amatneeks TM, Santos JC, Cavallet LHR, Michel RB. Qualidade de vida de pacientes em hemodiálise e sua relação com mortalidade, hospitalizações e má adesão ao tratamento. J Bras Nefrol. [Internet]. 2016 [acesso em 12 de ago 2021];38(4):411-20.

Doi.org/10.5935/0101-2800.20160066

12. Mello ML, Oliveira SS. Saúde, religião e cultura: um diálogo a partir das práticas afro-brasileiras. Saude soc. [Internet]. 2013 [acesso em $12 \mathrm{de}$ ago 2021]; 22 (4): 1024-35. Disponível em: https://www.arca.fiocruz.br/handle/ici $\mathrm{ct} / 27231$

13. Oliveira ERA de, Garcia ÁL, Gomes MJ, Bittar TO, Pereira AC. Gênero e qualidade de vida percebida: estudo com professores da área de saúde. Ciênc saúde coletiva. [Internet]. 2012 [acesso em 12 de ago 2021];17(3):741-7.

Doi.org/10.1590/S1413-

81232012000300021

14. Bravin AM, Trettene AS, Andrade LGM, Popim RC. Benefits of spirituality and/or religiosity in patients with chronic kidney disease: an integrative review. Rev Bras Enferm. [Internet]. 2019 [acesso em 12 de ago 2021]; 72 (2): 541-51. https://doi.org/10.1590/0034-71672018-0051
15. Fornazari SA, Ferreira RER. Religiosidade / espiritualidade em pacientes oncológicos: qualidade de vida e saúde. Psic: Teor e Pesq. [Internet]. 2010 [acesso em 12 de ago 2021]; $26 \quad$ (2): 265-72. Doi.org/10.1590/S010237722010000200008

16. Caires VG. Políticas para a educação profissional e a articulação entre o ensino profissional e o ensino médio: estudo comparativo sobre o ensino integrado em uma instituição da rede federal de educação profissional, científica e tecnológica de minas gerais. Trabalho \& Educação. [Internet]. 2016 [acesso em 12 de ago 2021]; 25(1) |331-333. Disponível em: https://periodicos.ufmg.br > article > download

17. Melo GAA, et al. Intervenção musical sobre a ansiedade e parâmetros vitais de pacientes renais crônicos: ensaio clínico randomizado. Rev. Latino-Am. Enfermagem. [Internet]. 2018 [acesso em 12 de ago 2021]; $26 \mathrm{e} 2978$. Doi.org/10.1590/15188345.2123.2978

18. Diniz AP, Minucci GS, Roama-Alves RJ, Souza e Souza LP. Espiritualidade e Religiosidade como práticas de enfrentamento ao uso abusivo de drogas. Rev Psi Divers Saúde. [Internet]. 2020 [acesso em 12 de ago 2021]; 9 (1): 88. Doi: 10.17267/23173394rpds.v9i1.2467

19. Gonçalves RMA, Lancman S, Sznelwar LI, Cordone NG, Barros J de O. Estudo do trabalho em Núcleos de Apoio à Saúde da Família (NASF), São Paulo, Brasil. Rev bras saúde ocup. [Internet]. 2015 [acesso em 14 de ago 2021]; 40(131): 59-74. Doi.org/10.1590/03037657000078013 
20. Grandizoli, MV. Depressão, desesperada, ideação suicida e qualidade de vida de pacientes em tratamento hemodialítico, 2019. Dissertação (mestrado)- Faculdade de Medicina de São João do Rio Preto. FAMERP. Programa de Pós Graduação em Psicologia e Saúde, Área de concentração: Psicologia e Saúde. Disponível em:https://bdtd.famerp.br/bitstream/te de/550/2/MarianaVidottiGrandizoli_D issert.pdf.

21. Souza FTZ, Oliveira JHA de. Sintomas Depressivos e Ansiosos no Paciente Renal Crônico em Tratamento Conservador. Revista Psicologia e Saúde. [Internet]. 2017 [acesso em 13 de ago 2021]; 17-31. Doi.org/10.20435/pssa.v9i3.429

22. Ventura CAA. Saúde mental e direitos humanos: o Brasil e a jurisdição da Corte Interamericana de Direitos Humanos. Int J Minor Group Rights. [Internet]. 2018 [acesso em 18 de ago 2021]; 25 (2): 236-60.

\section{Autor Correspondente ${ }^{\#}$}

Submissão: 2021-08-15

Aprovado: 2021-08-23 